\title{
Accuracy of single-pass whole-body computed tomography for detection of injuries in patients with major blunt trauma
}

\author{
Dirk Stengel MD PhD, Caspar Ottersbach BSc(Psych), Gerrit Matthes MD PhD, Moritz Weigeldt MD, \\ Simon Grundei MD, Grit Rademacher MD, Anja Tittel MD, Sven Mutze MD PhD, Axel Ekkernkamp MD PhD, \\ Matthias Frank MD, Uli Schmucker MD, Julia Seifert MD PhD
}

\begin{abstract}
- Abstract
Background: Contrast-enhanced whole-body computed tomography (also called "panscanning") is considered to be a conclusive diagnostic tool for major trauma. We sought to determine the accuracy of this method, focusing on the reliability of negative results.

Methods: Between July 2006 and December 2008, a total of 982 patients with suspected severe injuries underwent single-pass panscanning at a metropolitan trauma centre. The findings of the scan were independently evaluated by two reviewers who analyzed the injuries to five body regions and compared the results to a synopsis of hospital charts, subsequent imaging and interventional procedures. We calculated the sensitivity and specificity of the pan-scan for each body region, and we assessed the residual risk of missed injuries that required surgery or critical care.
\end{abstract}

Results: A total of 1756 injuries were detected in the 982 patients scanned. Of these, 360 patients had an Injury Severity Score greater than 15. The median length of follow-up was 39 (interquartile range 7-490) days, and 474 patients underwent a definitive reference test. The sensitivity of the initial pan-scan was $84.6 \%$ for head and neck injuries, $79.6 \%$ for facial injuries, $86.7 \%$ for thoracic injuries, $85.7 \%$ for abdominal injuries and $86.2 \%$ for pelvic injuries. Specificity was $98.9 \%$ for head and neck injuries, $99.1 \%$ for facial injuries, $98.9 \%$ for thoracic injuries, $97.5 \%$ for abdominal injuries and $99.8 \%$ for pelvic injuries. In total, 62 patients had 70 missed injuries, indicating a residual risk of $6.3 \%$ (95\% confidence interval $4.9 \%-8.0 \%$ ).

Interpretation: We found that the positive results of trauma pan-scans are conclusive but negative results require subsequent confirmation. The pan-scan algorithms reduce, but do not eliminate, the risk of missed injuries, and they should not replace close monitoring and clinical follow-up of patients with major trauma.
$\mathrm{S}$ evere trauma is one of the 10 leading causes of burden of disease in North America and Europe. ${ }^{1}$ According to a report from the Canadian National Trauma Registry, 14065 major injuries with an Injury Severity Score above 12 occurred between 2008 and 2009 in the eight provinces that contribute to the National Trauma Registry. ${ }^{2}$ Of these, there were 1605 (11\%) deaths, and 212098 hospital-days resulted.

Priority-oriented management requires accurate imaging during resuscitation to avoid missed injuries. ${ }^{3,4}$ Standardized algorithms, such as Advanced Trauma Life Support (ATLS), typically comprise physical examination, focused thoracoabdominal ultrasonography and plain radiography of the chest, spine and pelvis, followed by computed tomography (CT) of the head and other selected body areas. The effectiveness of this staged diagnostic approach, however, has been called into question in recent years..$^{5-9}$

Contrast-enhanced whole-body CT scanning, often referred to as "pan-scanning," was first proposed in the late 1990s as an alternative to sequential radiologic imaging in trauma settings. ${ }^{10}$ Pan-scan algorithms have been shown to accelerate diagnostic work-up, but their effect on survival is controversial. ${ }^{11-14}$ Opponents have voiced concerns about the overexposure of patients to radiation with the increasing and often uncritical use of CT scanning. ${ }^{15}$

We designed the PATRES (Pan-Scan for Trauma Resuscitation) study to assess the accuracy of the pan-scan in detecting injuries to different body regions in patients with suspected major blunt trauma.
Competing interests: Dirk Stengel is a board member of and a consultant for the German Trauma Association. He is a consultant, has provided expert testimony and received payment for lectures for the German Federal Statutory Accident Insurance, Biomet, DePuy, Stryker and the AO Foundation. He holds grants from these agencies, as well as from the Federal Ministry of Education and Research, and the European

Commission. Gerrit Matthes is a board member and has received payment for providing lectures from the German Advanced Trauma Life Support. He has received compensation for travel expenses from NATO for providing a lecture on trauma management. Axel Ekkernkamp is a consultant for Pfizer, Stryker and Novo Nordisk. He has provided expert testimony for DePuy, Rehavital and Medkon, and he has received payment for lectures from Johnson \& Johnson Medical, Depuy,

Medkon and Rehavital. None declared for Caspar Ottersbach, Moritz Weigeldt, Simon Grundei, Grit

Rademacher, Anja Tittel, Sven Mutze, Matthias Frank, Uli Schmucker and Julia Seifert.

This article has been peer reviewed.

Correspondence to: Dr. Dirk Stengel, dirk.stengel@ukb.de

CMAJ 2012. DOI:10.1503 /cmaj.111420 


\section{Methods}

\section{Study design and population}

In this study, we included data from 1000 consecutive patients with blunt trauma who were transferred directly from the scene to the emergency department of our institution, a metropolitan trauma centre, between July 2006 and November 2008. Resuscitation followed the ATLS algorithm (www.facs.org/trauma/atls) developed by the American College of Surgeons and involved a team of trauma and orthopedic surgeons, anesthesiologists and nursing staff.

We began implementing a pan-scan into the primary trauma survey in our institution in $1997,{ }^{16}$ and we continue to adapt our CT suite and scanning protocols in accordance with the latest multidetector technology.

According to the "red flag" criteria outlined in the German Trauma Association's clinical practice guidelines for the management of multiple trauma, ${ }^{17}$ a pan-scan was ordered in the following situations:

- if there was an injury mechanism that exposed the patient to a high risk of multiple trauma (i.e., a road traffic collision with presumed high-energy trauma, as evidenced by extrication or death of a car occupant, a pedestrian struck by a vehicle, or a fall from height)

- if a technical rescue was required

- if the patient had impaired physical or physiologic status (i.e., unconsciousness, intubation and ventilation, obvious signs of injury such as a bruise, hematoma, open wound or fracture, hemodynamic instability)

- if the suspicion of severe trauma was confirmed by paramedics or emergency doctors on scene.

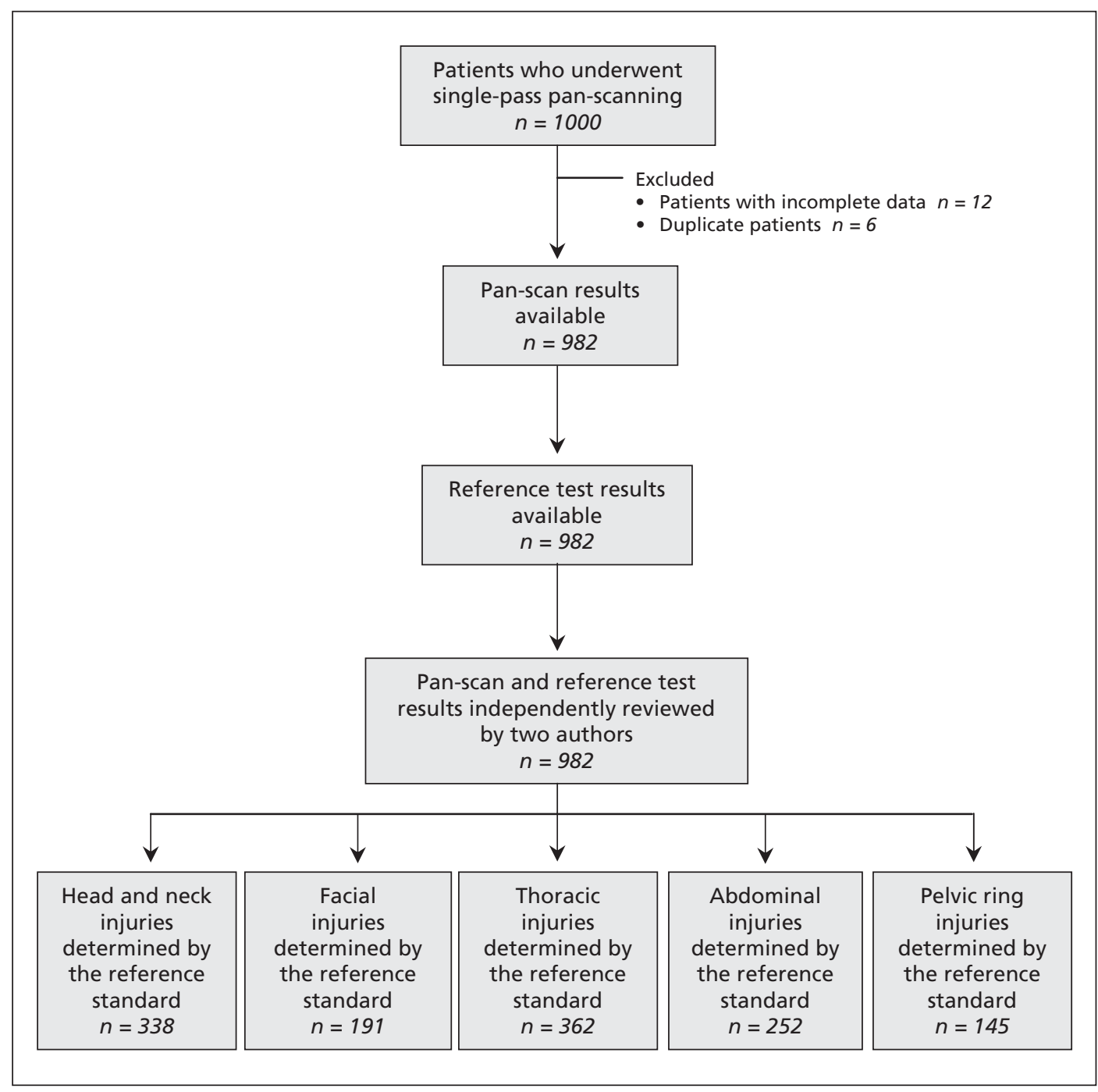

Figure 1: Flow diagram of the Pan-Scan for Trauma Resuscitation (PATRES) study of the accuracy of using single-pass whole-body computed tomography (pan-scan) to detect injuries in patients who had sustained high-velocity trauma. 


\section{Pan-scan protocol}

At our institution, the CT suite is adjacent to the trauma room. Patients are scheduled for a panscan during or after successful resuscitation if their physiologic parameters allow for the safe performance of whole-body CT imaging. During the study period, imaging was performed using a 64-slice multidetector CT scanner (Brilliance CT64, Philips, Cleveland, United States). The technical parameters of the protocol are described in Appendix 1 (available at www.cmaj.ca/lookup /suppl/doi:10.1503/cmaj.111420/-/DC1).

The images were read by the radiology consultant on call, and the results were immediately reported to the trauma team. All images were discussed the next morning by the radiologists and trauma and orthopedic surgeons during their daily conference.

For the purpose of this study, all images were independently reviewed a second time by two consultant radiologists to determine interobserver agreement.

\section{Definitions}

We defined the injuries in advance by consensus between radiologists, trauma and orthopedic surgeons and the methodologic supervisor (Appendix 2, available at www.cmaj.ca/lookup /suppl/doi:10.1503/cmaj.111420/-/DC1). We defined true-positive findings as injuries identified on initial pan-scans that were verified by repeated or further imaging or a certain intervention or

Table 1: Baseline characteristics and injury details of study patients

\begin{tabular}{|c|c|c|c|}
\hline Characteristic & $\begin{array}{c}\text { No. }(\%)^{*} \text { of } \\
\text { patients } \\
n=982\end{array}$ & Characteristic (continued) & $\begin{array}{c}\text { No. }(\%)^{*} \text { of } \\
\text { patients } \\
n=982\end{array}$ \\
\hline Age at injury, yr, mean (SD) & $42.0(19.4)$ & $\begin{array}{l}\text { Time between admission and scanning, min, } \\
\text { mean (SD) }\end{array}$ & $55.8(133.1)$ \\
\hline Sex, male & $730(74.3)$ & $\begin{array}{l}\text { Time between admission and scanning, min, } \\
\text { median (IQR) }\end{array}$ & $29(20-46)$ \\
\hline Covered by worker's compensation & $231(23.5)$ & Subsequent reference tests & \\
\hline Mechanism of injury & & Computed tomography & \\
\hline Road traffic injury $\dagger$ & $591(60.2)$ & Cranial & $257(26.2)$ \\
\hline Fall from height $\ddagger$ & $275(28.0)$ & Chest & $69(7.0)$ \\
\hline Fall on stairs & $54(5.5)$ & Abdominal & $72(7.3)$ \\
\hline Other§ & $62(6.3)$ & Pelvic & $47(4.8)$ \\
\hline ISSףl, mean (SD) & $14.1(13.0)$ & Spine & $112(11.4)$ \\
\hline ISS, median (IQR) & $10(4-21)$ & Ultrasound & $331(33.7)$ \\
\hline Multiple trauma (ISS >15) & $360(36.7)$ & Magnetic resonance imaging & $173(17.6)$ \\
\hline ISS, mean (SD) & $27.7(12.1)$ & Thoracotomy & $5(0.5)$ \\
\hline ISS, median (IQR) & $25(18-33)$ & Laparotomy & $37(3.8)$ \\
\hline Fracture of an extremity & & Plain radiographs & $711(72.4)$ \\
\hline Humeral shaft & $45(4.6)$ & Autopsy & $5(0.5)$ \\
\hline Forearm & $112(11.4)$ & Patients with any reference test & $816(83.1)$ \\
\hline Femoral shaft, any & $117(11.9)$ & Patients with $\geq 2$ reference tests & $522(53.2)$ \\
\hline Femoral shaft, bilateral & $29(3.0)$ & Patients with any conclusive reference test** & $474(48.3)$ \\
\hline Tibia & $169(17.2)$ & Duration of follow-up, days, mean (SD) & $293.7(420.7)$ \\
\hline Multiple, upper limb & $24(2.4)$ & Duration of follow-up, days, median (IQR) & $39(7-490)$ \\
\hline Multiple, lower limb & $78(7.9)$ & Death & $70(7.1)$ \\
\hline \multicolumn{4}{|c|}{$\begin{array}{l}\text { Note: IQR = interquartile range, ISS = Injury Severity Score, SD = standard deviation. } \\
\text { *Unless stated otherwise. } \\
\text { †Patients with road traffic injuries included car occupants }(n=260) \text {, motorcyclists }(n=140) \text {, pedestrians }(n=94) \text {, bicycle riders }(n=79) \text { and heavy-vehicle drivers } \\
(n=14) \text {. The injury mechanism was not specified in four cases. } \\
\text { ¥The mean height of falling was } 2.2 \text { (SD 2.4) metres. } \\
\text { §Other injuries: assaults }(n=14) \text {; equestrian injuries }(n=12) \text {; explosions }(n=9) \text {; airplane crashes and skydiving injuries }(n=8) \text {; burns }(n=8) \text {; crushes and spillages }(n \\
=8 \text { ); electric trauma }(n=3) \text {. } \\
\text { १The Injury Severity Score was computed as the sum of the squared six-point ordinal Abbreviated Injury Scale (AIS) severity code (known as the post-dot code of } \\
\text { the AIS) of the three most severe injuries located in the following anatomic regions: head and neck, face, chest, abdomen (including pelvic organ injuries), } \\
\text { extremities (including pelvic ring injuries), and external (i.e., skin and soft tissue). The individual AIS severity code can be traced from the AIS Codebook. }{ }^{21} \text { Although } \\
\text { the AIS may reach values between } 1 \text { (minor) and } 6 \text { (lethal), the ISS ranges from } 0 \text { to } 75 \text {. An ISS greater than } 15 \text { indicate the presence of multiple trauma. } \\
* * \text { Includes computed tomography or magnetic resonance imaging of any region, surgical intervention, or autopsy. }\end{array}$} \\
\hline
\end{tabular}




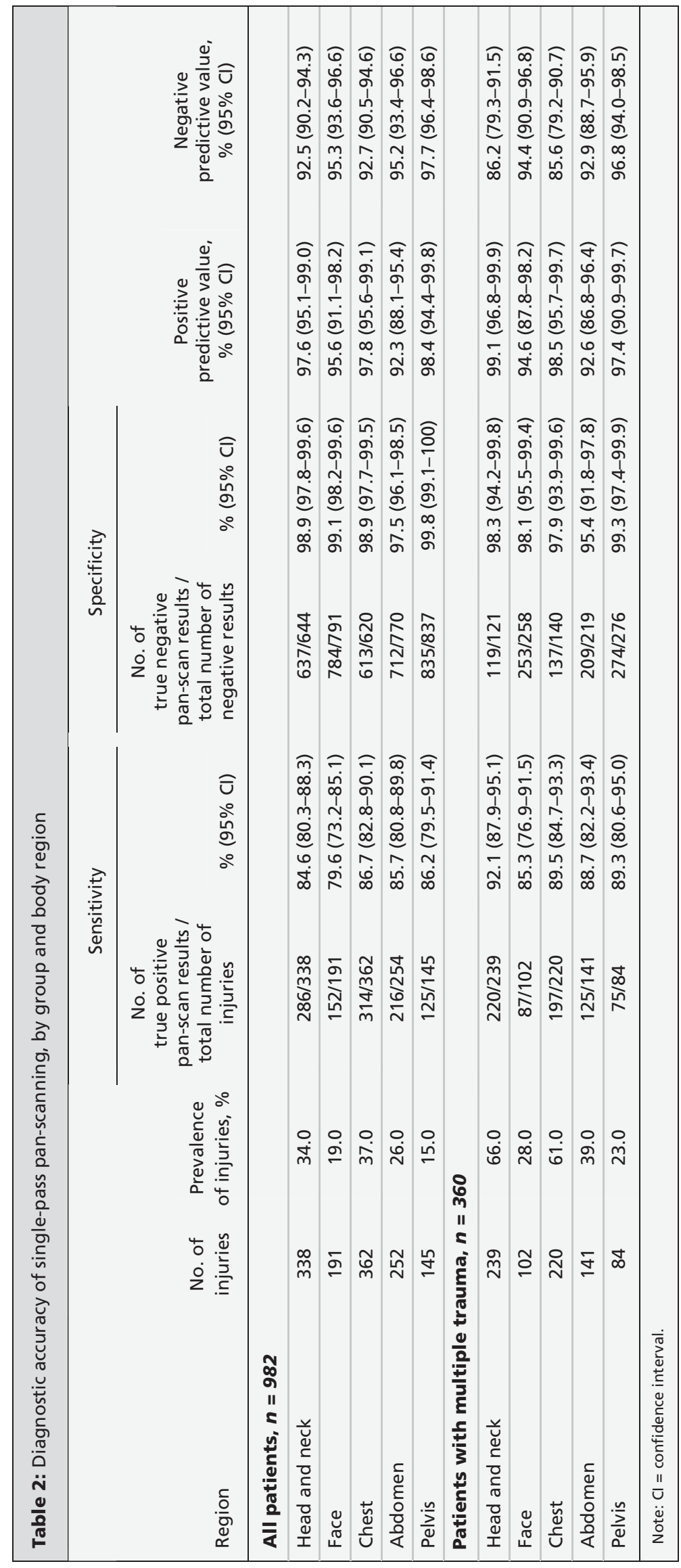

both, or as injuries that were documented in the hospital chart or final discharge report. We defined false-positive results as injuries that were suspected on the initial scans but could not be verified during the hospital course, either by intervention or radiologic imaging.

Any injury that was not detected by the panscan was classified as a false-negative result, regardless of its severity or therapeutic consequence. The absence of injuries in a predefined body region during both the initial and follow-up imaging or during clinical follow-up or both was classified as a true-negative result.

\section{Diagnostic reference standard}

Computed tomography is widely recognized as a diagnostic reference standard, which makes validating its accuracy with established methods difficult. In this study, we considered the most appropriate reference standard for comparing diagnostic accuracy to be all collected data pertaining to the progress and outcome (i.e., all clinical, radiologic and interventional data, and both in-hospital and outpatient follow-up data).

Our institution's clinical trial unit created a database with a prespecified set of injuries occurring to five body regions (i.e., head and neck, face, chest, abdomen, and pelvic ring) to facilitate and standardize data entry and documentation. These body regions are the same as those used in the calculation of the Injury Severity Score. Despite its limitations, the Injury Severity Score is the most common scoring system for anatomic injuries used worldwide. ${ }^{18,19}$ Injuries to two or more body regions and an Injury Severity Score greater than 15 indicates multiple trauma.

To enhance the validity of verifying the results of the index tests, two reviewers (M.W. and S.G.) independently scrutinized the electronic and paper charts of all included patients; they deliberately excluded the images and reports from the initial pan-scan. The charts included clinical and surgical notes, intraoperative findings, follow-up images, clinical follow-up and autopsy results. We obtained follow-up data, which included the latest contact with the patient in the outpatient or emergency department. In case of discrepancies, the reviewers reached consensus with a third reviewer (D.S.).

We also recorded injuries missed by the initial pan-scan that later required surgery, interventional procedures or monitoring in the intensive care unit.

\section{Ethics approval}

The study protocol was approved by the institutional review board of the Charité Universitätsmedizin Berlin, Berlin, Germany. A consent 
waiver was granted because we used routine hospital data. The study was preregistered in the International Standard Randomized Controlled Trial Number Register (ISRCTN41462125).

\section{Statistical analysis}

Descriptive statistics for continuous variables were expressed as mean, median, standard deviation (SD) and interquartile range (IQR). We expressed categorical variables as numbers and percentages. We assessed inter-rater agreement between radiology consultants for the index test and between clinical reviewers for the reference standard by use of Cohen's $\kappa .^{20}$

We calculated sensitivity, specificity and positive and negative predictive values by comparing the results of the index test (i.e., the results of the initial pan-scan) to the reference standard (i.e., the presence or absence of injuries based on the synopsis of all follow-up data). We calculated 95\% confidence intervals (CIs) for all measures of test accuracy.

We performed the analyses for each body region (head and neck, face, chest, abdomen, and pelvic ring) and for individual injuries in these regions.

We performed descriptive subgroup analyses for patients with and without multiple trauma (Injury Severity Score $>15$ and $\leq 15$, respectively) and for patients who underwent any conclusive reference testing (i.e., CT, magnetic resonance imaging, surgery or autopsy) or who were followed-up by clinical observation, radiography and ultrasonography alone. We also performed subgroup analyses by length of follow-up (separated into quartiles).

We graphically analyzed whether there was an optimal time window for scheduling patients for a pan-scan that is associated with highest sensitivity and specificity. The interval between arrival of the patient and the start of the scan was separated into centiles, and we computed diagnostic indices for cumulative numbers of patients undergoing the test within consecutive periods of time (e.g., $\leq 14 \mathrm{~min}$, $\leq 18 \mathrm{~min}, \leq 22 \mathrm{~min}$ ).

We used logistic regression analyses to model the association between the lag time to scanning and the incidence of therapeutically relevant missed injuries.

\section{Results}

Of the 1000 consecutive patients admitted and screened during the study period, we included 982 unique patients because of the availability of pan-scan reports and images. Table 1 presents the characteristics of all included patients. Figure 1 shows the study flowchart and the test verification protocol designed based on the Standards for the Reporting of Diagnostic Accuracy Studies. ${ }^{21}$ In total, a diagnosis of multiple trauma was made for 360 patients (36.7\%) based on the results of the reference standard.

The reviewers found that 77 (7.8\%) of the panscans were unnecessary or were a result of overtriaging. They concluded that selective scanning would have been sufficient for these patients. This included 40 patients with minor injuries (Injury Severity Score < 6), 22 patients with head and neck injuries, 11 patients with fractures of the mid-face, thoracic spine or lumbar spine and ribs, and 4 patients with complex fractures of the extremities.

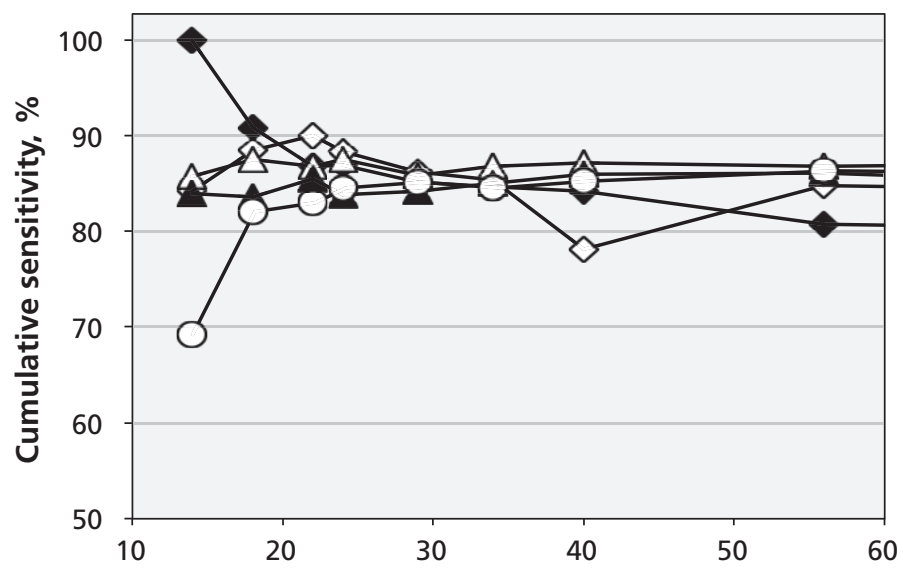

Time between admission and pan-scan, $\min$
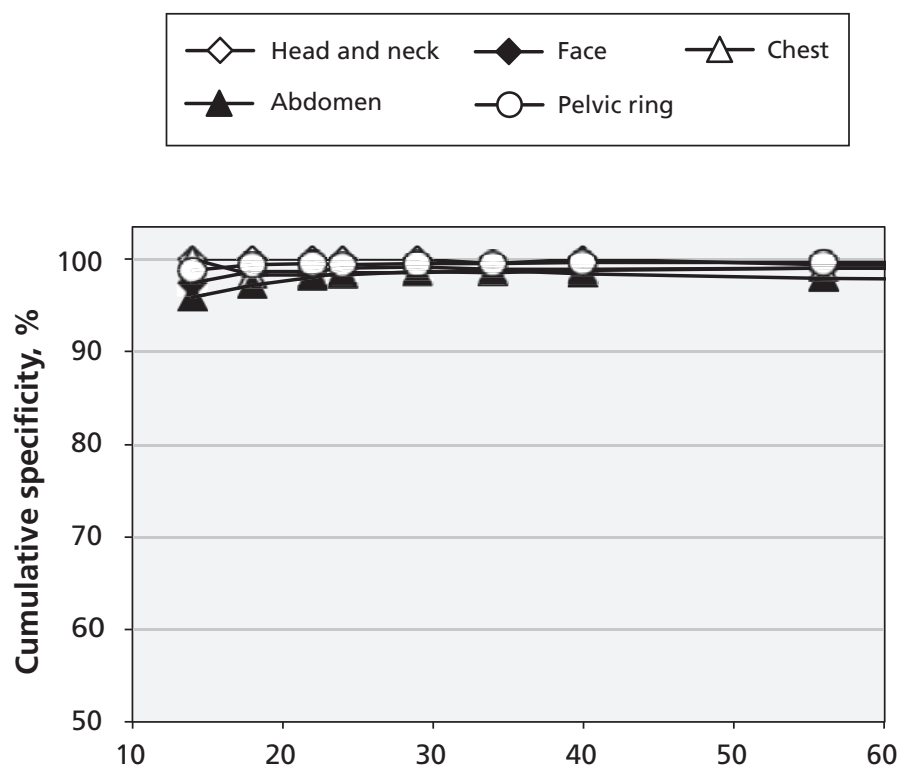

Time between admission and pan-scan, $\min$

Figure 2: Association between diagnostic accuracy and the time between admission to hospital and the start of the pan-scan. 
Table 3: Consequences of the 70 injuries (62 patients) that were missed by the initial pan-scan

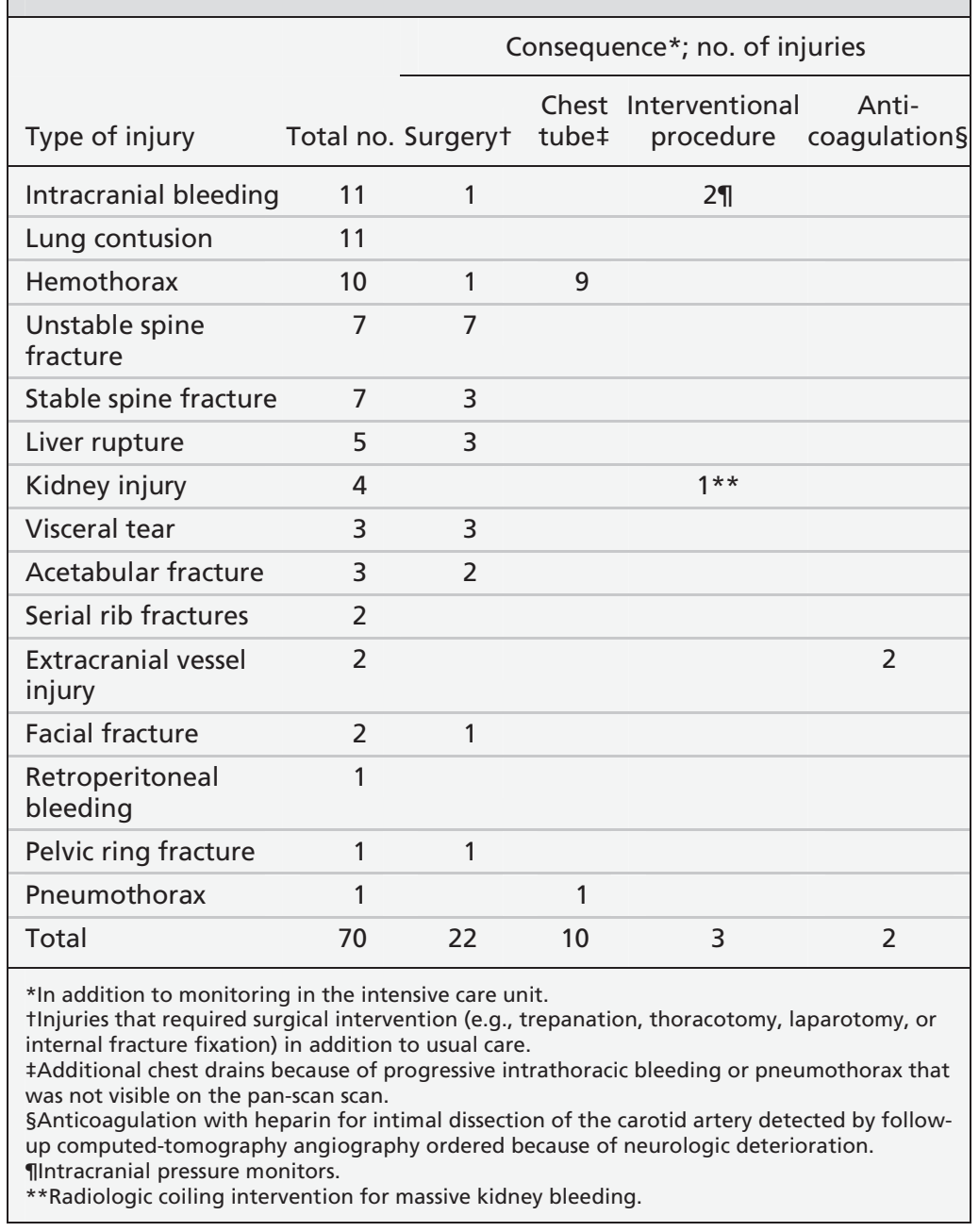

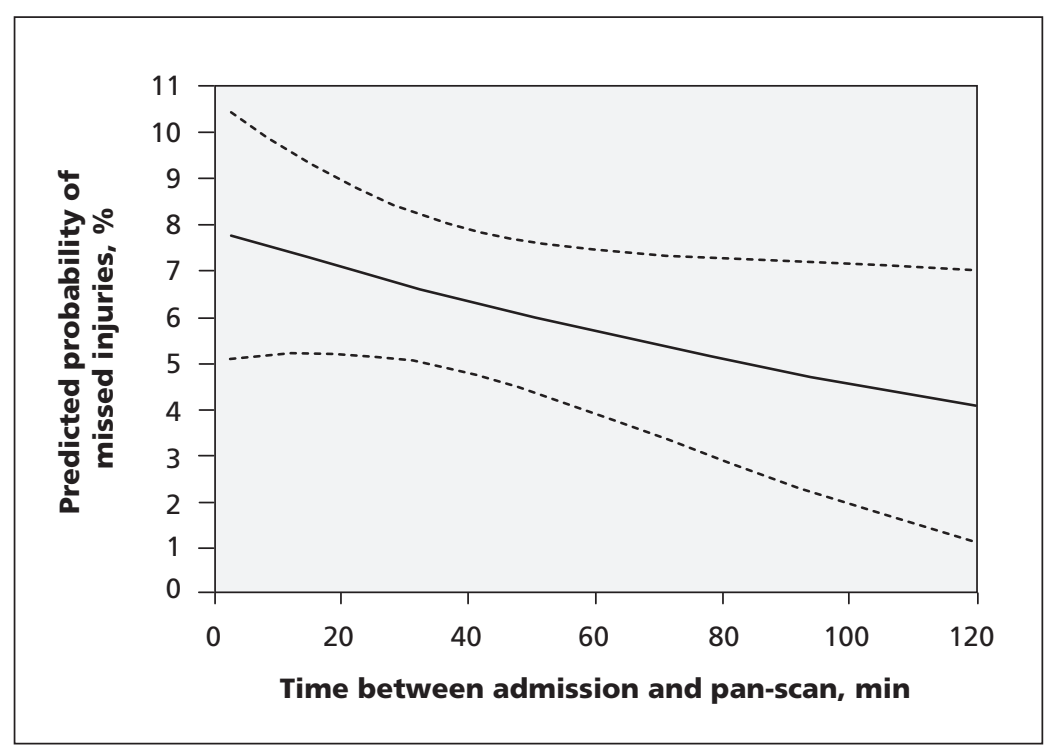

Figure 3: Predicted probability of missing therapeutically relevant injuries in relation to the time between admission to hospital and the start of the pan-scan, as determined by logistic regression. Dashed lines represent $95 \%$ confidence intervals.
Interobserver agreement between radiologists during re-reading of initial scans was substantial (median $\kappa$ 0.83, IQR 0.70-0.86). The reviewers agreed on the presence or absence of injuries with a median $\kappa$ of 0.88 (IQR 0.80-0.89).

Table 2 summarizes the diagnostic accuracy by body region for all patients and for the subgroup of patients with multiple trauma. The diagnostic accuracy for individual injuries is shown in Appendix 3 (available at www.cmaj.ca /lookup/suppl/doi:10.1503/cmaj.111420/-/DC1). The pan-scan was consistently specific $(97.5 \%$ $99.8 \%)$, but the sensitivity was variable $(79.6 \%$ $86.7 \%$ ) for detecting injuries to the body regions of interest. A higher sensitivity was found for patients with multiple trauma $(85.3 \%-92.1 \%)$ than for patients without multiple trauma.

Verification of the findings of the pan-scan by use of conclusive reference standards (Appendix 4, available at www.cmaj.ca/lookup /suppl/doi:10.1503/cmaj.111420/-/DC1) and the duration of follow-up (Appendix 5, available at www.cmaj.ca/lookup/suppl/doi:10.1503 /cmaj.111420/-/DC1) did not significantly influence the characteristics of the pan-scan.

Because of legal regulations, we did not have access to forensic autopsy reports. When we limited our analysis to the 912 surviving patients, we obtained virtually similar results (data not shown).

The optimal accuracy of the pan-scan was between 24 and 34 minutes after admission (Figure 2). During this period, the sensitivity was $83.8 \%-88.3 \%$ and specificity was $98.3 \%-100 \%$ for different body regions.

The initial pan-scan did not detect 70 injuries (in 62 patients) that subsequently required monitoring in the intensive care unit $(6.3 \%, 95 \% \mathrm{CI}$ 4.9\%-8.0\%, Table 3).

The predicted probability of missed injuries decreased slightly with longer intervals between admission and scanning (Figure 3). However, this trend was not significant (odds ratio 0.99, 95\% CI 0.98-1.00).

\section{Interpretation}

We found that single-pass whole-body computed tomography is highly specific but has variable sensitivity for the detection of injuries in patients with suspected blunt trauma. Screening tests in trauma are intended to immediately detect lifethreatening injuries. Given this premise, high specificity makes pan-scanning a valuable tool for priority-oriented treatment planning.

Negative results require confirmation during clinical monitoring or by follow-up examination and imaging. The main diagnostic gaps included injuries that demarcate after fluid resuscitation (e.g., solid organ contusions, 
mesenteric tears). We found the best balance between sensitivity and specificity when the pan-scan was performed about 30 minutes after admission. In addition to better visibility of lesions after blood circulation and tissue perfusion are restored, we speculate that the clinical situations that allowed a short delay before scanning were associated with less time pressure when reviewing the $\mathrm{CT}$ images.

This study of the diagnostic accuracy of panscanning contributes information to the primary, or efficacy, level of the health technology assessment cascade, and our results may help to understand the survival benefit associated with trauma pan-scanning observed in two previous studies. ${ }^{12,13}$ Injuries that can only be visualized by a whole-body scan may overemphasize the severity of the injury and artificially increase the ratio of observed to expected survivors. ${ }^{14,23}$ However, our data give support to the alternative theory that a primary pan-scan effectively omits many diagnostic steps between clinical suspicion and definitive proof of injuries that require immediate therapeutic attention.

\section{Limitations}

This was a single-centre study that has limited generalizability to other populations. The trauma algorithm established at our institution does not allow for a comparison of accuracy indices between pan-scans and conventional diagnostic work-ups. ${ }^{6-8,24}$ The imperfect reference standard used, which combined with clinical and radiologic tests, could have introduced partial verification bias,,$^{25}$ and we may have underestimated the sensitivity of the pan-scan results because our definitions of true and false results may have been too conservative. Finally, we did not address the problem of nontraumatic incidental findings, which occur in up to one-third of all patients who undergo trauma scanning. ${ }^{26}$

\section{Conclusion}

Positive pan-scan results are conclusive, but negative results require subsequent confirmation. Pan-scan algorithms reduce, but do not eliminate, the risk of missed injuries, and they should not replace close monitoring and clinical follow-up of patients with major trauma. Our results need to be validated in a prospective, multicentre setting using a uniform diagnostic reference standard. Further research is needed to determine the impact of pan-scan findings on management decisions..$^{27,28}$ New criteria for the appropriate application of pan-scans must be developed to accurately triage the patients with severe trauma who will benefit most from this diagnostic procedure.

\section{References}

1. Mathers CD, Loncar D. Projections of global mortality and burden of disease from 2002 to 2030. PLoS Med 2006;3:e442.

2. Canadian Institute for Health Information. National trauma registry 2011 report: hospitalizations for major injury in Canada, 2008-2009 Data. Montréal (Qc): The Institute: 2011.

3. Chen $\mathrm{CW}$, Chu CM, Yu WY, et al. Incidence rate and risk factors of missed injuries in major trauma patients. Accid Anal Prev 2011;43:823-8.

4. Pfeifer R, Pape HC. Missed injuries in trauma patients: A literature review. Patient Saf Surg 2008;2:20.

5. Deunk J, Brink M, Dekker HM, et al. Routine versus selective computed tomography of the abdomen, pelvis, and lumbar spine in blunt trauma: a prospective evaluation. J Trauma 2009;66: 1108-17.

6. Elmali M, Baydin A, Nural MS, et al. Lung parenchymal injury and its frequency in blunt thoracic trauma: the diagnostic value of chest radiography and thoracic CT. Diagn Interv Radiol 2007; 13:179-82.

7. Hashem R, Evans CC, Farrokhyar F, et al. Plain radiography does not add any clinically significant advantage to multidetector row computed tomography in diagnosing cervical spine injuries in blunt trauma patients. J Trauma 2009;66:423-8.

8. Hilty MP, Behrendt I, Benneker LM, et al. Pelvic radiography in ATLS algorithms: A diminishing role? World J Emerg Surg 2008; $3: 11$.

9. Stengel D, Bauwens K, Sehouli J, et al. Emergency ultrasoundbased algorithms for diagnosing blunt abdominal trauma. Cochrane Database Syst Rev 2005;CD004446.

10. Leidner B, Adiels M, Aspelin P, et al. Standardized CT examination of the multitraumatized patient. Eur Radiol 1998;8: 1630-8.

11. Sierink JC, Saltzherr TP, Reitsma JB, et al. Systematic review and meta-analysis of immediate total-body computed tomography compared with selective radiological imaging of injured patients. Br J Surg 2012;99:52-8.

12. Huber-Wagner S, Lefering R, Qvick LM, et al. Effect of wholebody CT during trauma resuscitation on survival: a retrospective, multicentre study. Lancet 2009;373:1455-61.

13. Hutter M, Woltmann A, Hierholzer C, et al. Association between a single-pass whole-body computed tomography policy and survival after blunt major trauma: a retrospective cohort study. Scand J Trauma Resusc Emerg Med 2011;19:73.

14. Stengel D, Frank M, Matthes G, et al. Primary pan-computed tomography for blunt multiple trauma: Can the whole be better than its parts? Injury 2009;40(Suppl 4):S36-46.

15. Snyder GE. Whole-body imaging in blunt multisystem trauma patients who were never examined. Ann Emerg Med 2008;52: $101-3$

16. Mutze S, Madeja C, Paris S, et al. Helical CT examination of multiple trauma patients in a digitized radiology department. Emerg Radiol 1999;6:77-80.

17. Deutsche Gesellschaft für Unfallchirurgie. S3-Leitlinie "Polytrauma / Schwerverletzten-Behandlung" (AWMF Register-Nr. 012/019). DGU Mitteil Nachr 2011;33:1-188.

18. Cameron PA, Palmer C. Developing consensus on injury coding. Injury 2011;42:10-1.

19. Lefering R. Trauma score systems for quality assessment. Eur J Trauma Emerg Surg 2002;28:52-63.

20. Landis JR, Koch GG. An application of hierarchical kappa-type statistics in the assessment of majority agreement among multiple observers. Biometrics 1977;33:363-74.

21. Gennarelli TA, Wodzin E. The abbreviated injury scale 2005. Update 2008. Des Plaines (IL): American Association of Automotive Medicine; 2008.

22. Bossuyt PM, Reitsma JB, Bruns DE, et al. Towards complete and accurate reporting of studies of diagnostic accuracy: the STARD initiative. BMJ 2003;326:41-4.

23. van Vugt R, Deunk J, Brink M, et al. Influence of routine computed tomography on predicted survival from blunt thoracoabdominal trauma. Eur J Trauma Emerg Surg 2011;37: 185-90.

24. Stengel D, Bauwens K, Rademacher G, et al. Association between compliance with methodological standards of diagnostic research and reported test accuracy: meta-analysis of focused assessment of US for trauma. Radiology 2005;236: 102-11.

25. Rutjes AW, Reitsma JB, Coomarasamy A, et al. Evaluation of diagnostic tests when there is no gold standard. A review of methods. Health Technol Assess 2007; 11:iii, ix-51.

26. van Vugt R, Dekker HM, Deunk J, et al. Incidental findings on routine thoracoabdominal computed tomography in blunt trauma patients. J Trauma 2011 Apr. 29 [Epub ahead of print] 


\section{RESEARCH}

27. Brink M, Deunk J, Dekker HM, et al. Added value of routine chest MDCT after blunt trauma: evaluation of additional findings and impact on patient management. AJR Am J Roentgenol 2008;190:1591-8.

28. Tillou A, Gupta M, Baraff LJ, et al. Is the use of pan-computed tomography for blunt trauma justified? A prospective evaluation. J Trauma 2009;67:779-87.

Affiliations: From the Centre for Clinical Research (Stengel, Ottersbach, Grundei), Department of Trauma and Orthopaedic Surgery (Matthes, Weigeldt, Seifert) and the Department of Radiology (Rademacher, Tittel, Mutze), Unfallkrankenhaus Berlin, Berlin, Germany; and the Department of Trauma and Reconstructive Surgery (Stengel, Ottersbach, Ekkernkamp, Frank, Schmucker, Seifert), Ernst Moritz Arndt University Medical Center, Greifswald, Germany.

Contributors: Gerrit Matthes and Caspar Ottersbach contributed equally to this work. Dirk Stengel, Gerrit Matthes, Grit Rademacher, Sven Mutze and Julia Seifert were responsible for the study conception and design. Caspar Ottersbach, Moritz Weigeldt and Simon Grundei were responsible for data collection and correlation of the index test and reference standard findings. Grit Rademacher, Anja Tittel and Sven Mutze collected and re-evaluated all radiologic images. Dirk Stengel and Caspar Ottersbach performed all statistical analyses, and Dirk Stengel drafted the manuscript. Dirk Stengel, Matthias Frank, Uli Schmucker, Axel Ekkernkamp, Gerrit Matthes and Julia Seifert critically revised the manuscript for important intellectual content. All authors read and approved the manuscript submitted for publication. Dirk Stengel is the guarantor of this study.

Funding: No third party funding in any form was obtained for data collection or analysis.

Acknowledgements: The authors thank Annette Luckmann, MA, Centre for Clinical Research, Unfallkrankenhaus Berlin, Berlin, Germany, for assistance with manuscript preparation. The authors thank Kathleen Füssler, MSc, Center for Clinical Research, Unfallkrankenhaus Berlin, Berlin, Germany, for assistance with data entry.

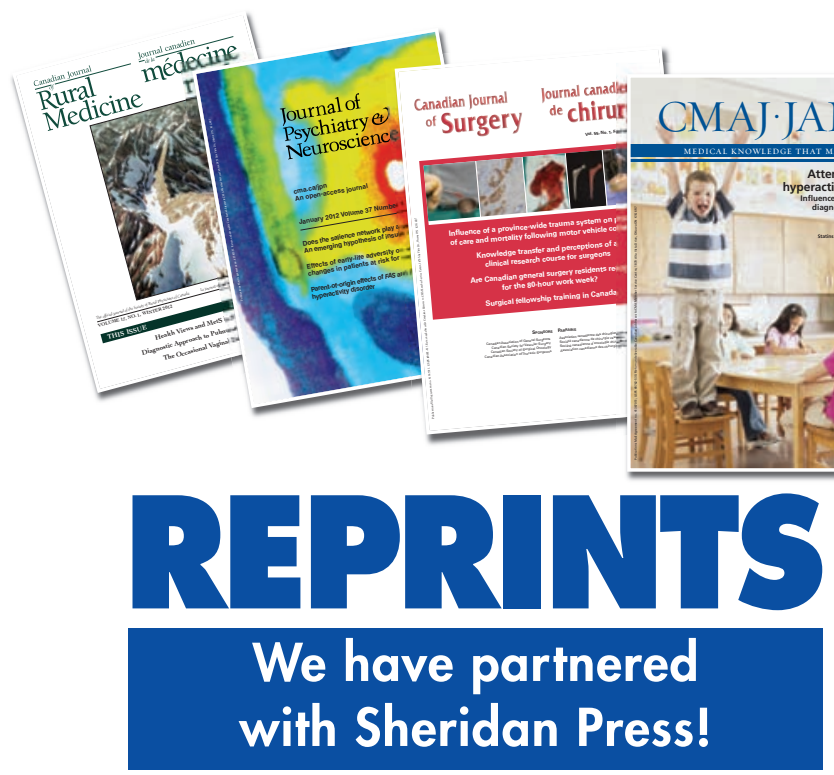

To purchase commercial article reprints and e-prints or to request a quote, please contact

\section{Matt Neiderer}

Content Sales

Sheridan Content Services

$800635-7181 \times 6265$

matt.neiderer@sheridan.com 